East African Medical Journal Vol. 81 No. 12 December 2004

CORONARY ARTERY DISEASE AND SYMPTOMS OF DEPRESSION IN A KENYAN POPULATION

F. G Nienga, FRCPsych, P.O. Box 73749, Nairobi, C0200, Kenya, C. G. Kamotho, BSc, MBChB, DTM\&H, P.O. Box 55650, Nairobi, 00200. Kenya M.D. Joshi, MBChB, MMed, MPH (Epi), Aga Khan Hospital, P.O. Box 30270, Nairobi, Kenya, D. K. Gikonyo, MBChB, MMed and M. Wanyoike. MBChB MMed. Nairobi Hospital, P.O. Box 30026, Nairobi, Kenya

Request for reprints to: Dr. F. G. Njenga, P.O. Box 73749, Nairobi, 00200, Kenya

\title{
CORONARY ARTERY DISEASE AND SYMPTOMS OF DEPRESSION IN A KENYAN POPULATION
}

\author{
F. G. NJENGA, C. G. KAMOTHO, M.D. JOSHI, D. K. GIKONYO and M. WANYOIKE
}

\begin{abstract}
Background: Depression and heart disease are replacing the traditional enemies of Africa such as infectious diseases and malnutrition as the increasing causes of disability and premature death. Little is known about the co-morbidity of heart disease and depression in Africa. Objective: To describe the prevalence of depression in Black Africans with and without Coronary Artery Disease as documented on coronary angiography at the Nairobi Hospital. Design: Prospective comparative study.

Setting: A private not for Profit 210 bed hospital, catering for fee paying middles class clintele. Results: Of the eighteen patients with an abnormal angiogram, the highest score on the BDI was 9 while the average was 2.11 . Of the seven with normal angiograms, the highest BDI was 5, and the average was 1.71. There was no statistical significance in these differences.

Conclusion: While African scientists must continue to concentrate on the urgent medical priorities of today (AIDS, malaria, measles, etc), cognisance has to be made of the other emerging epidemic, of the co-morbidity of coronary artery disease and depression. That no significant difference in depression score between the two groups was found could be due to a number of reasons including the small sample size achieved in this first study of its kind in Kenya.
\end{abstract}

\section{INTRODUCTION}

Dramatic changes in the overall health needs of the world's populations are occurring. It has been generally assumed that in the developing regions, where four-fifths of the world's people live, the leading causes of disease burden are communicable diseases. In fact non-communicable diseases such as depression and heart disease are replacing the traditional enemies such as infectious diseases and malnutrition as the leading causes of disability and premature death(1).

Little is known about the co-morbidity of heart disease and depression in Africa, while in the West there is increasing evidence that depression is associated with poor outcome in patients with coronary artery disease.

Psychological well-being is an important component of the health of an individual, and of the community at large. While a healthy state of mind is a key determinant in the enjoyment and prolongation of life, psychosocial stress can have adverse consequences on one's lifespan. In Swedish men, it was found that mental stress was associated with an increased risk of subsequent coronary heart disease (CHD) events(2). Also, in 12 Russian regions between 1989-1994, the greater the stress caused by major life events the greater the decline in male life expectancy(3).
There is evidence of the increasing incidence of coronary artery disease (CAD) in Africa. In two recent studies, Jablonski(4) and Schneider(5) writing from Kenya and Ethiopia respectively, allude to this increase. Similar trends have been reported from Japan and Australia.

The importance of psychosocial factors, in the development and expression of coronary artery disease has been debated extensively. Depressive symptoms are known to be associated with worse health status among patients with coronary artery disease. Cardiovascular disease is the leading cause of death and a major cause of morbidity and disability in the United States, with an estimated 6 million people having symptomatic CAD. It is estimated that the lifetime risk of suffering from depression is $20 \%$. Both depression and CAD are common and it would be expected that their cooccurrence would be high in the population.

The health status of cardiac patients should therefore include assessment and treatment of depression, since treatment of depression leads to improvement in health status, and is associated with better health outcomes. Treatment of depression may improve cardiovascular outcomes among patients with coronary disease.

The one month community-based prevalence of major depressive episodes is about 5\%. Among CAD patients, however, the prevalence of major depression 
is about 3-fold higher. Recent epidemiological studies evaluating the relationship between depression and CAD among healthy and CAD populations consistently demonstrate a significant prospective relationship between the occurrence of major depressive episodes and the incidence of cardiac events(3). Depression is present in over $45 \%$ of patients admitted to hospital after a myocardial infarction and is an independent risk factor for increased mortality and morbidity after myocardial infarction. The risk persists regardless of smoking status, deprivation score, and presence of diabetes or hypertension(5).

It is plausible that depression has a pathogenic role for ischaemic heart disease and at least six possible explanations have been proposed. Firstly, depression may lead to coronary events directly or indirectly via poorer health behaviours, such as increased smoking or decreased activity(7). This has been shown in patients who are depressed after myocardial infarction (MI)(8) and the same mechanism could operate before infarction. Such behaviour changes may lead to a poorer cardiovascular risk profile - for example, higher cholesterol concentration or blood pressure. Secondly, the association between depression and the risk of heart disease may be due to and effect of tricyclic antidepressant drugs. Thirdly the depression has been shown to be proarrythmogenic in patients with established ischaemic heart disease(9). This is thought to be due to changes in the balance between sympathetic and parasympathetic nerve activity - for example an increase in sympathetic nerve activity or a decrease in parasympathetic nerve activity or both of these. This mechanism might operate in depressed patients without established ischaemic heart disease, increasing their risk of developing it or accelerating its onset. Fourthly, depression might result in an unfavourable lipid profile resulting from an interaction between the catecholamine and steroid axes(10). Fifthly, depression might be confused with "vital exhaustion" - the prodromal symptoms of tiredness, apathetic mood and sadness which occur immediately before an M1. Finally, there may be a separate and yet unidentified, aetiological factor that causes both depression and ischaemic heart disease (8).

Attempts have been made to establish the physiological/biochemical link between cardiac events and depression. Recent studies have linked the inflammatory process, immune system, hypercoagulability, stress, and depression.

The immune system for example, plays a role in the progression of coronary artery diseases and its clinical manifestations as acute coronary syndromes. It is well established that psychological factors can act as risk factors for acute coronary syndromes(11). Chronic stress may be involved in the conversion of a stable artherosclerotic plague into an unstable one leading to myocardial infarction, following its rupture(11).
It is against this background of the link between depression and CAD that the present study was conceived, as part of a broader study for the satisfaction for the MMed thesis of one of the authors (CGK).

Since the setting up of the Nairobi Hospital Cardiac Catheterisation Laboratory in 1996, numerous hitherto inaccessible and/or non-affordable cardiac diagnostic and therapeutic interventions, including coronary angiography, have been made available to Kenyans. Coronary angiography is currently the gold standard diagnostic test for Coronary Artery Disease. There is no data on the local population relating depressive illness with CAD, especially using the coronary angiogram (CA).

\section{OBJECTIVES}

The aim of the study was to analyse the relationship of depressive symptoms with presence of CAD in Black Africans while the specific objectives were i) To describe the prevalence of depressive symptoms in Black Africans with CAD as documented on coronary angiography at the Nairobi Hospital catheterisation laboratory ii) To describe the prevalence of depressive symptoms in Black Africans with normal coronary arteries as documented on CA at the catheterisation laboratory iii) To compare the prevalence of depressive symptoms between the two groups. The study sought to identify any relationship between a traditional marker of depressive illness, namely the Beck Depression Inventory (BDI), and presence or not of CAD as documented on $\mathrm{CA}$ in Black Africans in Kenya.

\section{MATERIALS AND METHODS}

Every morning the list of names of patients who had been booked for a coronary angiogram were obtained from the catheterisation laboratory of the Nairobi Hospital, together with the name of the referring cardiologist or clinic. The patients were interviewed before or after the procedure, either at their respective clinics or at the Nairobi Hospital, and were invited to take part in the study. After informed consent. an investigator-administered questionnaire was completed. Diagnoses and other data were obtained from the hospital and clinic notes.

The inclusion criteria were for one to have undergone a coronary angiogram at the Nairobi Hospital catheterisation laboratory Black Race; Consent - by the cardiologist to interview his patient, and informed consent by the patient for the interview. The exclusion criteria were Non-Black race; Age less than 18 years of age and refusal to give consent.

The BDI has been used recently in the groundbreaking; study on the effects of treating depression after myocardial infarction. It is a 13 -item self report measure of severity of depressive symptoms with total scores between $\mathrm{O}$ and 39 ; a score of 13 or higher is the threshold for considering clinical depression measures above 26 indicate severe depression on this scale.

While other markers of depressive illness do exist, the Beck Depression Inventory is the most widely used locally and internationally. Moreover, as demonstrated by a pilot 
study, inclusion of other tests would make the questionnaire unduly long for the patient.

The results of the CA (normal / abnormal) were noted. Abnormal was taken as $>1=50 \%$ stenosis on one or more of the coronary arteries, judged by the standard method for interpreting the presence and severity of stenosis in the epicardial coronary arteries, which continues to be visual assessment or "eyeballing"(12).

Siudy design: This was a cross sectional study.

Ethical approval: Approval for the study was obtained from the Educational Committee of the Nairobi Hospital. Informed consent was obtained from the patients according to the Helsinki Declaration. All data of the patients was treated with utmost confidentiality at all stages of the study.

\section{RESULTS}

In the six-month study period between October 2002 and March 2003, 62 patients underwent coronary angiograms at the Nairobi Hospital catheterisation laboratory. Twenty-nine were black, and four did not give consent. Data was thus eventually collected for 25 patients. Of these, 18 had CAD, seven were normal. Of the 18 patients with $C A D$, the mean age was 60.83 years with a range of 4380 years. Of the seven with normal angiograms the mean age was 58.71 with a range of 47 to 68 . With regard to age, no statistically significant difference was found.

All the 17 males were married, while all but two females were married, with one each being widowed and separated. This was a well educated group with 13 of the males and two of the females having a University degree; two males and one female had achieved secondary education, one male and three females had only primary education. One female had no formal education; another had undergone non-University tertiary education. Data was missing for one male.

Physical activity was varied among those with CAD two did no physical exercise, three did it only in the past, the rest all did regular aerobic exercise. Among the seven with normal coronary arteries two did no physical exercise, one exercised in the past, the remaining four were on regular exercise.

Smoking: Of those with CAD, none were current smokers, four were former smokers, fourteen never smoked. Of those with normal angiogram, none were current smokers, five were former smokers, and two had never smoked. Of the eighteen patients with an abnormal angiogram, the highest score on the BDI was 9 while the average was 2.11. Of the seven with normal angiograms, the highest BDI was 5, and the average was 1.71 .

Data analysis: This categorical data was converted into percentages, with the corresponding $95 \%$ confidence intervals. Comparison of categorical data was done using the Chi-square test or Fischer's exact test. Statistical significance was defined as a two-tailed $p$ value of less than or equal to 0.05 . Statistical analysis did not show any difference between the two groups in the Beck scores.

\section{DISCUSSION}

The results show a non-significant difference between the Beck scores of the patients in the two CA groups. None of the patients in the study had evidence of a depressive illness, with a maximum Beck Score of 9 (normal). This negative finding is itself interesting since studies from the West show that depression may be an independent risk factor for ischaemic heart disease(8). Moreover among patients with coronary disease, depressive symptoms are strongly associated with patient-reported poor health status(13). The Heart and Soul Study in a recent large prospective cohort study that found depressive symptoms in $20 \%$ of patients with coronary artery disease(13).

Participants with depressive symptoms were more likely than those without depressive symptoms to report at least mild symptom burden. Their results suggest that depressive symptoms are an important factor in the perceived health status of patients with coronary disease, their results suggest that efforts to improve the health status of cardiac patients should include assessment and treatment of depressive illness.

Other non-cardiac factors that impinge on the mortality and morbidity in patients with CAD include levels of perceived social support. Recent studies have shown that depression and low perceived social support (LPSS) are associated with increased cardiac morbidity and mortality in CHD patients(14). Depression in cardiac patients is associated with significant psychological, social and physical disability and its effective treatment enhances quality of life and improves overall functioning(14).

Failure to recognise and treat the psychiatric complications of myocardial infarction (Ml) may aggravatc the underlying cardiac condition and interfere with its treatment and prognosis.

Since major depression affects about one in five patients in the weeks after an acute myocardial infarction(5) and is associated with an increased risk of cardiac morbidity and mortality, there is considerable interest in the question of whether treating depression will improve medical prognosis in these patients.

Persons who are depressed and who have preexisting cardiovascular disease have a 3.5 times greater risk of death than patients who are not depressed and have cardiovascular disease. Increased susceptibility to platelet activation has been proposed as one of the mechanisms by which depression acts as a significant risk factor for thrombotic events(16).

There is an indication of enhanced platelet activation and aggregation in depressed patients. Patients with a depressive disorder show signs of a hyperactive platelet 5-HT(2A) receptor signal transduction system as measured by increased platelet calcium mobilization after stimulation of platelets with serotonin(15). 
There is also evidence that depressed post infarction patients are more often re-admitted for cardiar reasons and their work resumption is delayed. Further, there are indications that depression and myocardial infarction have an aetiological relationship via the behaviour and emotions that are a risk factor for the development of both disorders. Depression and myocardial infarction are both stress-related disorders. A pathological stress reaction is seen in both conditions and triggers immune activation, enhanced blood cortisol levels, disturbance of serotonin metabolism and increased sympathetic activation(17).

The destruction of the World Trade Centre and associated terrorist activities of September 11, 2001 have spurred interest in understanding the medical consequences of terrorist activity. Since the World Trade Centre attack was associated with a rise in posttraumatic stress syndrome and affective disorders afterwards, there is accordingly interest in both the acute and more prolonged health effects that could be engendered following terrorist attacks. Known pathophysiological effects of acute stress, whether produced in a laboratory environment or by studying naturally occurring acute stressors, include: the induction or potentiation of cardiac arrhythmias; the induction of myocardial ischaemia in susceptible patients with underlying coronary artery discase; acute increases in arterial blood pressure with its ability to cause shear stress; the precipitation of worsening endothelial function and/or endothelial injury; coagulation abnormalities; and haemoconcentration. There are other non-cardiac causes of complications. A high level of anger has a powerful effect on the incidence of preventable cardiovascular death. Persuasive clinical evidence indicates that anger evokes physiological responses that are potentially lifethreatening in the setting of CAD. When angina is associated with periods of emotional stress or anger, the angina is not usually a result of progressive coronary disease, but rather is due to an increase in oxygen demand, while job strain, high Job demands, and, to some extent, low decision latitude, are associated with an increased risk of CAD among British civil servants(18). It seems that the relationship between depression and coronary artery disease is complex but indisputable.

Lauzon was able to show that depressed patients were more likely to undergo cardiac catheterisation and were more likely to undergo percutaneous coronary intervention within 30 days of first admission to hospital. Patients with depression on admission had higher rates of a composite of cardiac complications, including recurrent ischaemia, infarction or congestive heart failure during their first stay in hospital or re-admission for angina, recurrent acute myocardial infarction, congestive heart failure or arrhythmia, compared with patients who were not depressed on admission(19). Additionally, a self-reported history of treatment for depression is independently associated with subsequent myorcardial infarction in treated hypertensive patients without prior cardiovascular disease.

Our study is interesting for a number of reasons. Firstly, it is the only study from Africa that addresses two hitherto unrecognised common disorders that could cost the continent dearly if ignored. Secondly, it raises important questions for Africans and the rest of the world. In general, it is acknowledged that African systems of social support are still largely intact. If social support is better in Africa than in the West, does it explain the relative absence of depression in patients with coronary disease?

Another interesting issue raised by our study relates to the possibility that CAD by itself, before complicated by ischaemia does not cause depression. In that case, would treatment of depression in people known to have CAD lower the incidence of depression after myocardial ischaemia? Since stress has been associated with precipitation of myocardial infarction, what protective strategies exist in African societies?

\section{CONCLUSION}

Africa is a traumatised continent, ravaged by natural and manmade disasters $(20)$, while poverty is an independent risk factor for depression. These two stresses augur badly for people in Africa at risk of coronary artery disease. While African scientists must continue to concentrate on the urgent medical priorities of today (AIDS, malaria, measles, etc), cognisance has to be made of the other new and emerging epidemic of the co-morbidity of coronary artery disease and depression.

The fact that none of these patients, at high risk for depression, was found to be suffering from the disease, suggests a relatively low prevalence of depression in the population. We know however that this is not to be the case. Other explanations must be found.

The patients examined in this study using the Beck Depression Inventory may have suffered from depression previously and had now recovered spontaneously. It is possible therefore that this type of study should be complimented by a full psychiatric examination including a catalogue of past psychiatric illnesses. Following up this cohort could on the other hand establish a link between the onset of depressive symptoms and myocardial infaretion. Even more interesting would be to follow-up depressed and non-depressed survivors of myocardial infarction in Africa. It is hoped that this study will stimulate thought and research in this field. Limitations: Several limitations were inherent in this study.

Selection bias: This was the principal limitation of this study. The selection of the patients was on the basis of a CA. All those patients referred for a CA, including those who eventually had normal studies, were patients at high risk for coronary artery disease. It is probable therefore that depression was selected out because it 
is not readily recognised as a risk factor. The small numbers in the study would have confounded this error. The criteria for referral for CA was not standardised in this naturalistic study in which each cardiologist sent patients for $\mathrm{CA}$ using criteria that may have been different from other practices.

The target population was the Black African population in Kenya, the sample studied was select and non-random, mainly from the middle and upper sectors of society. The potential for introduction of bias was therefore significant.

Though not representative of the entire Black population in Kenya, the sample under study served as a surrogate marker. The results would still contribute to a better understanding of the presence of depressive illness in patients undergoing $\mathrm{CA}$ in Kenya.

Measurement bias: This applied in the evaluation of the actual coronary angiograms and the subsequent classification as normal, or abnormal. The coronary angiogram remains the gold standard diagnostic test for the evaluation of epicardial atherosclerotic coronary artery disease. The standard method of interpreting the severity of stenosis continues to be visual assessment or "eyeballing."(2). The subjective measurement bias thus introduced would apply equally to both groups studied, such that it did not affect the comparison results. The high technical quality of the facility contributed to better and reproducible assessment of the CA. The investigator, who was present during the angiogram procedures, was able to observe an acceptable uniformity in interpretation by the various cardiologists.

\section{REFERENCES}

1. Jenkins, R., McCulloch, A. Friedli, L. and Parker, C. The public health burden of mental illness. Developing a national mental health policy. Maudsley Monograph No. 43; Psychology Press London. Pg 15.

2. Rosengren. A., Tibblin, G. and Wilhelmsen L. Selfperceived psychological stress and incidence of coronary artery disease in middle-aged men. Amer. J. Cardio. 1991; 68: $1171-1175$.

3. Evans, R.G., Barer, M.L. and Marmor, T. Why are some people healthy and others not? The determinants of health of populations. New York, Aldine de Gruyter. 1994

4. Jablonski-Coben, M.S.. Kosgei, R.J., Rerimoi, A.J. and Mamlin, J.J. The emerging problem of coronary heart disease in Kenya. East Afr. Med. J. 2003; 80:293-297
5. Schneider, J. and Bezabih, K. Causes of sudden death in Addis Ababa, Ethiopia. Ethiop Med. J. 2001; 39:323-340.

6. Hippisley-Cox, J., Fielding, K. and Pringle, M. Depression as a risk factor for ischemic heart disease in men: population based case-control study. Brit. Med. J. 1998; 316:1714-1719

7. Carney, R.M., Rich, M.W.. Freedland, K.E. el al. Major depressive disorder predicts cardiac events in patients with coronary artery disease. Psychosom Med. 1998; 50:627-633.

8. Ladwig, K.H., Roll. G., Breithardt, G., Budde, T. and Borggrefe, M. Post-infarction depression and incomplete recovery 6 months after acute myocardial infarction. Lancet. 1994; 343:20-23.

9. Frasure-Smith, N., Lesperance, F. and Talajic, M. Depression and 18-month prognosis after myocardial infarction. Circulation. 1995; 91:999-1005.

10. Wassertheil-Smoller, S. Applegate, W.B., Berge, K. et al. Change in depression as a precursor of cardiovascular events. Arch. Intern. Med. 1996; 156:553-561.

11. Kop, W.J. The integration of cardiovascular behavioural medicine and psychoneuroimmunology: new developments based on converging research fields. Brain Behav. Immun. 2003; 17:233-237.

12. Pompa, J.J., Bittl, J.A. and Levin, D.C. Coronary arteriography and intravascular ultrasonography. In: Braunwald (Ed) Heart Disease, 6th Ed 200 l.

13. Ruo, B., Rumsfeld, J.S, Hiatky, M.A. et al. Depressive symptoms and health-related quality of life. The Heart and Soul Study. J. Amer. Med. Ass. 2003; 290:2:215-221

14. Writing Committee for the ENRICHD Investigators. Effects of treating depression and low perceived social support on clinical events after myocardial infarction. $J$. Amer. Med. Ass. 2003; 289:23:3106-3116.

15. Carney, R. E., Freedland, K. E., Veith, R.C. and Jaffe. A S. can treating depression reduce mortality after an acute myocardial infarction? Psychosom. Med. 1999: 61:666-675.

16. Schins, A., Honig, A. Criins, H. Baur, L. and Hamulyak, K Increased coronary events depressed cardiovascular patients: 5-HT(2A) Receptor as missing link? Psychosom. Med. 2003; 65:729-737.

17. Honig, A. Depression following a heart infarct and increased risk of death. Ned Tijdschr Geneeskd. 2000; 144:1307-1310.

18. Kuper, H. and Marmot. M. Job strain, job demands, decision latitude, and risk of coronary heart disease within the Whitehall 11 study. J. Epidemiol Comm. Health. 2003; 57:147-153.

19. Lauzon, C., Beck, C.A.. Huynh, T. et al. Depression and prognosis following hospital admission because of acute myocardial infarction. CMAJ. 2003; 168:547-552.

20. Njenga, F. G., Kigamwa. P. and Okonji, M. Africa: the traumatised continent, a continent with hope. Int. J. Psychiat. 2003: 1:4-7. 